\title{
Philosophiques
}

\section{Constructivisme, anti-essentialisme et racisme}

\section{Nestor Capdevilla}

Volume 40, numéro 2, automne 2013

URI : https://id.erudit.org/iderudit/1023706ar

DOI : https://doi.org/10.7202/1023706ar

Aller au sommaire du numéro

Éditeur(s)

Société de philosophie du Québec

ISSN

0316-2923 (imprimé)

1492-1391 (numérique)

Découvrir la revue

Citer ce document

Capdevilla, N. (2013). Constructivisme, anti-essentialisme et racisme.

Philosophiques, 40(2), 461-464. https://doi.org/10.7202/1023706ar d'utilisation que vous pouvez consulter en ligne.

https://apropos.erudit.org/fr/usagers/politique-dutilisation/ 


\title{
Constructivisme, anti-essentialisme et racisme
}

\author{
NESTOR CAPDEVILA \\ Département de philosophie, Université Paris Ouest Nanterre La Défense \\ n.capdevila@wanadoo.fr
}

Le livre de Magali Bessone propose une défense puissante et convaincante d'une conception constructiviste du concept de race et de son objet dans une perspective antiraciste. Elle s'oppose d'abord aux antiracistes qui fondent leur combat sur l'absence de contenu biologique. Les classes existent parce qu'elles sont socialement construites. Du coup, elle s'oppose également au raciste qui croit à leur substrat biologique. Il faut donc utiliser le terme de race pour décrire une société où la discrimination raciale existe, et la combattre.

Les questions de l'étendue de cet usage légitime, de ses conditions concrètes et de son pouvoir critique restent cependant posées. Le livre est écrit en pensant au contexte français (p. 193) alors que la littérature utilisée et discutée est largement anglo-saxonne. Ce décalage est principalement expliqué par un aveuglement du républicanisme français qui frappe d'interdit le mot «race». Mais cela veut également dire que les différences historiquesont un effet sur notre manière de penser: ce qui est désigné comme «race» dans un pays est nommé "nation", "nationalité, "ethnie ", "groupe ethnique ", dans d'autres (p. I3). Le contexte américain est "paradigmatique» (p. 204) pour la race. "Ce n'est pas la culture noire que méprise le raciste, mais les Noirs » remarque Appiah (p. 202). On pourrait même interroger les limites de cette distinction de la culture et de la race. La valorisation antiraciste du jazz a souvent racialisé ce qui semble être un produit essentiellement culturel. Il est une musique noire, inséparable de l'histoire et de la condition présente des Noirs aux États-Unis ${ }^{1}$ qui, selon une dialectique souvent décrite, n'a pu être acceptée par les Blancs qu'en étant édulcorée ou "blanchie». Quand elle aborde le contexte français, le vocabulaire de Magali Bessone est plus souple. Le républicanisme a permis que les immigrés soient discriminés «sur la base de l'affiliation raciale ou religieuse"; il a perpétué le racisme et les inégalités en refusant de reconnaitre "la dimension ethnique des relations sociales» (p. 219 $)^{2}$. Ces formulations suggèrent que les mots «nation", "nationalité,

1. «Les œuvres de Bessie étaient aussi totalement «sales» que celles de Robert Johnson ou de Memphis Minnie. Pourquoi ? parce que tous étaient victimes de la même expérience, telle est la base, la source de la musique noire: une douleur, une blessure, que chaque homme, chaque enfant, ressent dans ses tripes. C'est une douleur inexplicable qui n'a pas besoin d'explication pour les initiés, mais qui est vue comme quelque chose d'étrange et de lascif à ceux qui en sont indemnes" (Archie Shepp,cité par Christian Béthune, Adorno et le jazz. Analyse d'un déni esthétique, Paris, Klienscksick, 2003, p. 52).

2. "Traiter la "question raciale" en la réduisant à et en la déplaçant sur la "question de l'immigration" qui est en réalité celle des relations interethniques et du racisme du point de vue 
«ethnie », "groupe ethnique» qui sont souvent des euphémismes ne le sont pas toujours. Quand cessent-ils de l'être ? La référence à la religion est le meilleur indice de la difficulté. Si une masse de «Français de souche» se convertissaient à l'islam le plus rigoriste, plus ou moins théocratique, comment réagirait la République? Si la conversion ne suscitait aucune difficulté, alors les problèmes actuels apparaîtraient comme raciaux. S'ils persistaient, la question pourrait-elle être traitée en termes raciaux?

L'équivoque n'est pas due au hasard, car ce qui importe pour le racisme biologique n'est pas la biologie pure, mais ce qui dans la biologie est censé avoir une portée non biologique, indésirable pour la vie sociale, morale, culturelle, religieuse, etc. C'est pourquoi les termes non raciaux ne sont pas nécessairement des euphémismes. Ils peuvent aussi toucher à l'essentiel. Il en résulte également que la race peut devenir un euphémisme. La conquête de l'Amérique (p. 29) relève de la définition donnée du racisme (p. I78-I84), et les Indiens sont une race au sens constructiviste (p. 87). Elle est néanmoins un exemple de "racisme sans race" qu'il est discutable de penser en termes de races, même si l'on est prêt à faire un usage rétrospectif du terme à partir du moment où il touche un point crucial. Ce vocabulaire occulte, comme un euphémisme, le scandaleux pouvoir discriminant de l'humanisme. Le journal de Colomb témoigne d'une évidence de l'humanitée ${ }^{3}$ confirmée par la bulle de donation d'Alexandre VI qui charge les rois catholiques de l'évangélisation. Le sommet juridique de la violence hispanique est une sommation, le requerimiento, qui déduit l'unité de la loi et l'unicité du gouvernement de l'humanité à partir de l'unité du genre humain pensée de manière monogéniste. Les Indiens sont des barbares et des infidèles, mais cela veut dire qu'ils sont opprimés en tant qu'hommes, pour leur incapacité à vivre, comme ils le devraient, en accord avec leur nature. Il n'est pas absurde de se demander avec Cornélius de Pauw (même s'il sacrifie au préjugé que l'appartenance de l'Indien au genre humain avait été problématique) s' $\mathrm{s}^{\prime}$ il aurait mieux valu persister dans l'opinion que les Américains étaient des singes, que de les reconnaître pour des hommes, et de s'arroger le droit affreux de les assassiner au nom de Dieu ${ }^{4}$.

L'expérience imaginaire de la conversion paraît très improbable, car on ne voit pas ce qui pourrait pousser des Français à se convertir à une religion que la tradition chrétienne a combattue et qui, par sa tendance théocratique

de la République $[\ldots] »($ p. 220).

3. Par exemple: "Ils doivent être bons serviteurs et industrieux (de buen ingenio) parce que je vois que très vite ils répètent tout ce que je leur ai dit, et je crois qu'aisément ils se feraient chrétiens, car il ne m'a paru qu'ils n'étaient d'aucune secte. S'il plaît à Notre Seigneur, au moment de mon départ, j'en emmènerai d'ici à six à Vos Altesses pour qu'ils apprennent notre langue. Je n'ai vu dans cette île aucune bête d'aucune sorte sauf des perroquets " (La déconverte de l'Amérique. I, Journal de bord, 1492-1493), Paris, Éditions de La Découverte, I99 I, p. 6I).

4. Cité par Michèle Duchet, Anthropologie et histoire an siècle des Lumières, Paris, Albin Michel, 1995, p. 207. 
et antiféministe, menace des acquis fondamentaux de la République. Ce jugement peut être justifié dans une conception substantialiste, et même racialisée, de la nation, ou dans une conception constructiviste. La contingence inscrite dans le constructivisme permet a priori de penser une semblable conversion. Il n'en reste pas moins vrai que la nation s'est effectivement construite de manière à la rendre très improbable. Cette convergence est suffisamment forte pour interroger le sens et la portée critique de l'optique constructiviste. En quoi exactement s'oppose-t-elle au racisme et au républicanisme? Magali Bessone donne cette définition constructiviste de la race: «Les races désignent des agrégats d'individus partageant des propriétés superficielles qui servent de critère pour une pratique sociale ou une ascription, ou elles désignent des groupes de personnes produits causalement ou institutionnellement par une telle pratique sociale» (p. 87). Le républicain antiraciste estimera peut-être que cette définition est trop éloignée de ce qu'on entend ordinairement par race pour qu'il soit réellement question de la race. Lorsqu'on lit cette définition, est-il possible de deviner que son objet est la race? Ne pourrait-elle pas s'appliquer aux sorcières, voire à la nation ? Tout dépend sans doute des propriétés superficielles et de leur traitement. La question est alors de savoir si l'on peut faire abstraction de la définition ordinaire de la race, principalement biologique. Faut-il inclure la fausse conscience raciste de la race dans la vraie conscience constructiviste de la race? L'inconvénient de ce surcroît de précision est de se mettre sous la dépendance du raciste au moment où on aiguise son arme contre lui. Le conservatisme du constructivisme est sensible quand le constructiviste, qui affirme l'existence des races, est confronté au cas du passing (p. IO8-III). Peut-il dire à quelle race appartient "objectivement» Susie Philips? Peut-il faire autre chose que constater qu'elle est blanche dans un pays, noire dans un autre, ou ni blanche ni noire dans une société sans races, blanche ou noire dans un sens totalement neutre dans une société devenue indifférente à ces questions de race $^{5}$ (peut-être réduites à des décisions individuelles comme la religion aujourd'hui), ou d'une couleur encore inconnue dans une société future racialement reconstruite? Le constructivisme décrit le conflit entre les forces qui luttent pour (re)définir les races ou les dépasser. Peut-il donner des clefs pour résoudre le conflit dans telle ou telle direction?

On peut même se demander si le raciste ne pourrait pas s'accommoder du constructivisme. Considérons cette déclarationde Condorcet: "Quelle que soit la cause qui les a rendus [les esclaves noirs] incapables d'être hommes, ce

5. «Une société idéale est une société sans races, «indifférente aux différences» raciales ", où il n'y a aucun sens à penser en termes de catégorisations raciales » (p. I I). Remarquons qu'il n'est pas contradictoire de parler d'égalité des races. Voir l'argumentation de l'Haïtien Anténor Firmin dans le cadre du paradigme anthropologique raciste du XIX ${ }^{\mathrm{e}}$ siècle, De l'égalité des races humaines (anthropologie positive), Paris, I 885 , réédité chez L'Harmattan en 2003. 
que le législateur leur doit, c'est moins de leur rendre des droits que d'assurer leur bien-être ${ }^{6}$.» Il est frappant de voir que cette proposition raciste est indifférente à la cause de l'infériorité du Noir. Qu'elle soit due à la biologie, au climat, à la mauvaise éducation, à l'oppression ou à l'exploitation, en un mot qu'elle soit naturelle ou construite, le résultat est le même, une incapacité à être libre. On peut juger ce fait très regrettable et même souffrir de ne pouvoir accorder plus de droits à ces êtres malheureusement frappés par la nature, le destin ou l'histoire. Le fait est là, et nous devons y adapter nos exigences morales. "Ce n'est pas manquer de respect que d'asservir celui qui n'a malheureusement pas la capacité d'être réellement libre» n'ont cessé de dire les racistes humanitaires depuis Sepúlveda.

Certes, le constructivisme souligne, contre le raciste biologique, le caractère contingent et déconstructible de la race. Mais le raciste sait aussi que les races peuvent changer en bien ou en mal. Il met précisément en œuvre des politiques pour neutraliser les mauvaises races (en les exterminant, en les isolant, voire en les dissolvant ${ }^{7}$ ), pour empêcher la dégénérescence des bonnes, favoriser leur amélioration, et parfois même celles des races inférieures comme le colonialiste humanitaire. Le raciste peut accepter le constructivisme si le résultat contingent a une identité et une solidité suffisantes pour qu'il se sente substantiellement et durablement menacé. Or c'est précisément ce que lui apprend le constructiviste. En dénonçant le caractère construit de l'objet que le raciste prend pour naturel, il met involontairement en relief la capacité des constructions à fonctionner durablement comme des natures: «Les races sont toujours là! Elles sont contingentes, construites et déconstructibles? Très bien! Reconstruisons-les à notre avantagedans un néo-néoracisme! ", peut-il se dire. Comme le remarque Magali Bessone, «le risque d'essentialisme est présent dans tous les termes qui fonctionnent comme substitut de celui de race» (p. I6). Le constructivisme est anti-essentialiste, mais son objectivité, qui légitime le recours au terme de race, a l'inconvénient d'enregistrer le processus d'essentialisation ou de naturalisation. Un usage raciste du constructivisme semble a priori possible. L'est-il réellement? Comment le constructivisme peut-il le prévenir?

6. Cité par Louis Sala-Molins, Le code noir ou le calvaire de Canaan, Paris, PUF, I987, p. $272-273$.

7. «Pour étendre notre agriculture, il serait nécessaire d'hispaniser nos Indiens. Leur oisiveté, leur sottise et leur indifférence envers les tâches normales laissent penser qu'ils sont issus d'une race dégénérée qui se dégrade à mesure qu'elle s'éloigne des origines [...]. Il serait fort souhaitable que les Indiens s'éteignissent par métissage avec les Blancs, en les libérant du tribut et d'autres charges et en leur accordant la propriété privée de la terre » (Pedro Fermín cité par Benedict Anderson, L'imaginaire national, Paris, La Découverte, I996, p. 27). 